\title{
Pour en finir avec les Monomanes de Géricault : considérons leur rôle dans la construction du mythe de l'artiste
}

\section{Ginette Jubinville}

Volume 41, numéro 1, 2016

URI : https://id.erudit.org/iderudit/1037555ar

DOI : https://doi.org/10.7202/1037555ar

\section{Aller au sommaire du numéro}

\section{Éditeur(s)}

UAAC-AAUC (University Art Association of Canada | Association d'art des universités du Canada)

\section{ISSN}

0315-9906 (imprimé)

1918-4778 (numérique)

Découvrir la revue

Citer cet article

Jubinville, G. (2016). Pour en finir avec les Monomanes de Géricault :

considérons leur rôle dans la construction du mythe de l'artiste. RACAR : Revue

d'art canadienne / Canadian Art Review, 41(1), 62-75.

https://doi.org/10.7202/1037555ar
Résumé de l'article

Théodore Géricault's Monomanes (Portraits of the Insane ; 1820s) were rediscovered in 1863, just four years before his first biography was published. This article examines this previously unstudied conjuncture in order to put to rest some of the myths surrounding these works and to show that their circulation contributed to the discourse on Géricault that was taking shape at the time. These five portraits have been described as displaying a duality that places them between art and psychiatry, between science and popular culture. This article argues that this resulted from the amalgamation of two distinct understandings of monomania : the scientific one at the time of their production and the popular one at the time of their rediscovery.
Tous droits réservés (C) UAAC-AAUC (University Art Association of Canada | Association d'art des universités du Canada), 2016
Ce document est protégé par la loi sur le droit d'auteur. L'utilisation des services d'Érudit (y compris la reproduction) est assujettie à sa politique d'utilisation que vous pouvez consulter en ligne.

https://apropos.erudit.org/fr/usagers/politique-dutilisation/ 


\title{
Pour en finir avec les Monomanes de Géricault: considérons leur rôle dans la construction du mythe de l'artiste
}

\author{
Ginette Jubinville
}

\begin{abstract}
Théodore Géricault's Monomanes (Portraits of the Insane; 1820s) were rediscovered in 1863 , just four years before his first biography was published. This article examines this previously unstudied conjuncture in order to put to rest some of the myths surrounding these works and to show that their circulation contributed to the discourse on Géricault that was taking shape at the time. These five portraits have been described as displaying a duality that places them between art and psychiatry, between science and popular culture. This article argues that this resulted from the amalgamation of two distinct understandings of monomania: the scientific one at the time of their production and the popular one at the time of their rediscovery.
\end{abstract}

Ginette Jubinville est chargée de cours à l'Université de Montréal. Elle est l'auteure de «Le portrait de la monomanie: rencontre de subjectivités» publié dans RACAR au printemps 2011

-jubinville.ginette@gmail.com

1. Les commentateurs de ces œuvres sont très nombreux mais peu ont apporté un éclairage complètement nouveau sur elles. Je ne retiendrai donc que les sources qui ont réellement avancé des éléments de preuves historiques pour soutenir leurs thèses, la pièce historique originale étant la lettre de Louis Viardot, publiée par Charles Blanc dans «La Chronique des Arts et de la Curiosité», Supplément à la Gazette des Beaux-Arts en 1864, dont il sera largement question dans cet article. Les informations de ce seul document historique proviennent du Dr Lachèze, propriétaire des œuvres en 1863 lors

Bien des questions ont été posées, par différents spécialistes, aux Monomanes de Géricault: sont-ils des portraits ou des études physiognomoniques? Ont-ils été créés en réponse à une commande pour un psychiatre ou constituent-ils un projet personnel de Géricault? Enfin, représentent-ils ou non des aliénés? Toutes ces questions ont donné lieu à une foule d'interprétations, de suppositions et de débats. En fait, malgré tous les écrits érudits qui portent sur ces œuvres, elles conservent leur mystère. Et si les questions soulevées ont donné lieu à une quantité de réponses, aucune ne s'avère complètement satisfaisante.

Alors, pourquoi écrire un nouveau texte sur le sujet? Pour mettre en évidence le seul point qui puisse être éclairci de façon concrète sur ces œuvres vu l'état actuel de la recherche: ${ }^{1}$ c'est-à-dire cerner comment leur redécouverte en 1863 a contribué à enrichir la légende et la gloire de Géricault, au moment même où, quarante ans après son décès, de nombreux auteurs se penchaient sur son œuvre et sur sa vie. En effet, durant les années 1860, la mise en place d'un mythe entourant l'artiste, qui allait s'avérer tenace, était en branle. Il construisait une image de Géricault comme artiste précurseur du romantisme, issu de la tradition classique, mais qui en subvertissait les conventions.

Le mythe de Géricault est construit en particulier par son biographe-hagiographe, Charles Clément. C'est, en effet, chez ce dernier que les commentateurs subséquents se documenteront. Sa monographie sur l'artiste paraît en $1867,{ }^{2}$ mais se base sur des recherches qui avaient été commencées dès 1860-

de leur redécouverte, qui affirme tenir ces tableaux et les détails des faits historiques de leur premier propriétaire, le Dr Georget, disciple d'Esquirol, un des pères de la psychiatrie française. Charles Clément, le premier biographe de Géricault les inscrit dans son catalogue raisonné de 1867 (réédité en 1879) et il reprend tous les éléments de cette lettre sans les questionner (Charles Clément, Géricault: étude biographique et critique, avec le catalogue raisonnéde l'œuvre du maître, $3^{\mathrm{e}}$ éd., augm. d'un suppl. et ornée de 30 planches, Paris, 1879). L'article publié en 1941 de Margaret Miller correspond à la première étude spécialisée sur ces portraits en lien avec la psychiatrie naissante française (Margaret Miller, «Géricault's Paintings of the Insane», Journal of the Warburg and Courtauld Institutes, vol. 4, no $3 / 4,1941$, p. 151-163). Denise Aimé-Azam, dans son ouvrage original de 1956 parle pour la première fois des liens possibles à tirer entre la production des œuvres et l'état mental de Géricault souffrant d'épisodes de dépression nerveuse dans les années 1819-1820. Ce serait doncà cette occasion que Géricault aurait consulté le Dr Georget (Denise Aimé-Azam, Géricault: l'énigme du peintre de la Méduse, Paris, 1983). Lorenz Eitner, qui produit une importante monographie sur Géricault en 1983, tire lui aussi des liens entre les œuvres et Georget, Esquirol et le domaine de la psychiatrie française. Il est aussi celui qui attire l'attention sur les antécédents de maladie mentale dans la famille de Géricault (Lorenz Eitner, Géricault: sa vie, son œuvre, Paris, 1991). Albert Boime dans un article original de 1991, avance une théorie nouvelle, à savoir que Géricault a été utilisé par Georget pour analyser de manière extensive ces malades désormais disponibles au regard scrutateur du psychiatre, répondant ainsi à la propre monomanie du commanditaire (Albert Boime, «Portraying 
Monomaniacs to Service the Alienist's Monomania: Gericault and Georget», Oxford Art Journal, vol. 14, $n^{\circ} 1,1991$, p. 79-91. Toutes ces références historiques sont corroborées dans la revue de littérature de la thèse de Rita Goodman qui porte sur ces œuvres spécifiques (Rita Goodman, Théodore Géricault's Portraits of the Insane: Art, Psychiatry and the Politics of Philanthropy, University of Michigan, 1996). Pour Goodman ces œuvres sont à inscrire, comme celles de Géricault qui se penchent sur la traite des noirs, l'abolition de l'esclavage ou le scandale du naufrage de la Méduse, comme mettant de l'avant la politique libérale de la Restauration des Bourbons. La position qu'elle soutient en regard du lien avec la psychiatrie est que si la commande a été initiée par un aliéniste, ce serait plutôt de la part d'Esquirol, le maître de Georget.

Tous les autres commentateurs des Monomanes les interrogent sous divers angles et selon les préoccupations historiques, formelles ou critiques de chacun. II reste à relever, comme le souligne encore Goodman (op. cit., p. 39), qu'étonnamment les auteurs ne questionnent pas le témoignage de Lachèze, à l'exception de Margaret Miller (op. cit.) ; Régis Michel dans le catalogue de l'exposition de 1991 (Paris: Éditions de la Réunion des musées nationaux); Thomas (row (Emulation: Making Artists for Revolutionary France, New Haven, 1995); et Brendan Prendeville, «The Features of Insanity, as Seen by Géricault and by Büchner», Oxford Art Journal, vol. $18, n^{\circ} 1,1995$, p. 96-115.

Enfin, l'événement de grande envergure le plus récent à s'être penché sur ces œuvres est l'exposition intitulée Géricault, la folie d'un monde qui s'est tenue du 21 avril au 31 juillet 2006, au Musée des Beauxarts de Lyon. Bruno Chenique, le spécialiste actuel de Géricault et commissaire général de l'exposition, avec Sylvie Ramond, directrice du musée de Lyon et commissaire de l'exposition, ont articulé cette exposition autour d'une œuvre dont le musée est propriétaire, la Monomane de l'envie. Dans le dossier de presse qui présente le catalogue de l'exposition, ils expriment l'angle selon lequel ils abordent ces cinq tableaux dont trois sont exposés. L'exposition vise à démontrer qu'ils ne sont pas à lire comme des témoins de la psychiatrie naissante, mais comme une réflexion politique se penchant sur la marginalité (www.mba-lyon.fr/static/ mba/contenu/pdf/presse/dp-gericault.pdf).

2. Clément, op. cit.
$1862,{ }^{3}$ précisément dans les mêmes années où la série des Monomanes refait son apparition. Cetarticle se penche sur la concordance de dates qui n'a, jusqu'à présent, pas été relevée et qui apparaît pourtant très significative. Elle permet en effet d'éclipser certains mystères entourant les œuvres et d'établir comment leur diffusion a pu contribuer à renforcer le discours qui s'établissaitalors autour de Géricault, le désignant comme l'artiste de l'histoire contemporaine et celui de l'humanité souffrante exprimée dans un réalisme quotidien. L'argument principal de ce texte repose sur le constat que c'est par une fusion de deux conceptions historiques de la monomanie que ces œuvres endossent ce rôle.

En effet, au moment de la réalisation des Monomanes, dans les années 1820, le concept même de monomanie est alors le fer de lance d'une nouvelle spécialisation médicale, la psychiatrie, qui fait la fierté de la nation française. Cette maladie est alors comprise comme un délire partiel qui se caractérise par l'apparence rationnelle, raisonnable, normale, du malade qui ne présente qu'une obsession spécifique. Progressivement au cours du siècle, ce concept se transforme radicalement pour devenir, dans les années 1860, une catégorie culturelle bénigne, affectant des citoyens issus du commun, présentant des obsessions banales et quotidiennes. En donnant aux cinq figures peintes par Géricault la désignation de monomanes et en leur assignant des délires spécifiques-jeu, vol, vol d'enfant, envie, commandement militaire-ses premiers récepteurs leur ont conféré, pour toute leur histoire subséquente, cette dualité, scientifique et populaire, qui en est venue à les définir.

Les seuls éléments connus, à ce jour, qui lient les Monomanes de Géricault au domaine de la psychiatrie française proviennent d'une lettre du critique d'art et marchand Louis Viardot adressée à Charles Blanc, historien de l'art et fondateur de la Gazette des Beaux-arts. La lettre est publiée dans ladite Gazette du 3 janvier $1864 .{ }^{4}$ Viardot y décrit cinq œuvres peintes qu'il reconnaît comme étant de Géricault. Il les appelle Cinq études d'aliénés et explique qu'il vient de les découvrir à Baden-Baden dans le grenier de son ami, le Dr Lachèze, qui les aurait achetées du Dr Étienne-Jean Georget (1795-1828), un contemporain de Géricault. Il s'agit d'œuvres qui ont été oubliées depuis près de quarante ans et que Viardot désigne comme des portraits d'aliénés. D'après lui, des détails concrets saisis par l'artiste les individualisent, comme la coiffe défaite, le regard hardi de la Monomane de l'envie, le bleu du regard et la moue du Monomane du vol, la béquille (ou la canne) de la Monomane du Jeu, le numéro 121 visible sur la médaille du Monomane du commandement militaire, et le vêtement soigné, bourgeois du Monomane du vol. Ce sont des portraits en buste, les personnages sont vus de face et les têtes, quasiment de grandeur nature se présentent de trois quarts. Les regards sont dirigés vers un point à l'extérieur du cadre et le rapport avec le spectateur se fait par cette «attention individualisée dans une direction particulière $»^{5}$ qui donne au récepteur la conscience de l'espace dans lequel ils sont représentés, un espace qui l'inclut. Viardot note que les sujets portent des vêtements usuels et non des tenues d'internement. Ce sont des vêtements d'hiver, des vêtements de sortie, qui connotent une vie publique. Les modèles sont immobiles malgré l'effet créé par la position oblique du corps et du regard qui suggère un mouvement imminent. L'éclairage latéral en clair-obscur dessine leurs traits et les ombres portées indiquent des sources lumineuses différentes. Le corps et le vêtement, brossés à grands traits de pinceaux avec 
3. Pour préparer son ouvrage qui fera office de bible de Géricault pendant de longues années, Clément puise ses sources chez ceux qui ont déjà écrit sur l'artiste: Louis Batissier, Géricault, Rouen, Extrait de la Revue du XIXe siècle, s. d., [1841]; Jules Michelet, Géricault, L'enfant du siècle, Caen, 1848; Gustave Planche, Géricault, Paris, 1851; Ernest Chesneau, Le Mouvement moderneen peinture. Géricault, Paris, 1861

4. Louis Viardot, «La Chronique des Arts et de la Curiosité», Supplément à la Gazette des BeauxArts, 1864, Paris: tome deuxième.

5. Comme Alois Riegl le décrit en parlant des personnages de la Ronde de Nuit de Rembrandt, dans Le portrait de groupe hollandais (Paris, 2008), p. 369

6. Rita Goodman fait état de ce fait dans sa thèse précitée. Elle relate (op. cit., p. 44, n 74) que le testament manuscrit de Georget (Archives nationales, LIV 1327, du $1^{\text {er }}$ mai 1828) ne fait aucune mention de ces œuvres, pas plus qu'elles ne sont désignées dans l'inventaire après décès de Georget (Archives nationales, LIV 1827). Régis Michel aussi avait relevé ce fait (Régis Michel, Géricault: l'invention du réel, Paris, 1992). des empâtements qui marquent une facture rapide, se confondent presque avec le fond sombre du tableau qui ne signale d'aucune manière le lieu où ils sont représentés. Par contre, les traits du visage sont plus travaillés et présentent des coups de pinceaux plus précis. Ces œuvres en camaïeux de tons de terre présentent toutes une touche de blanc lumineux autour du visage qui y dirige l'attention.

La lettre qui présente les Monomanes dans la Gazette pourrait sembler une source irréfutable. Cependant, les faits qui y sont présentés, que Viardot puise dans les souvenirs du Dr Lachèze, comportent suffisamment d'erreurs pour susciter le doute sur la justesse et la véracité de l'ensemble des informations qu'elle contient. Par exemple, l'auteur nomme erronément le Dr Georget médecin-chef de la Salpêtrière (l'hôpital parisien pour les femmes aliénées) alors qu'il n'y a été qu'interne pendant quelques années; il se trompe sur les dates de naissance et de mort de ce dernier; il en fait l'ami d'enfance de Géricault même si quatre ans les séparent et qu'aucun autre document, aucun témoignage fiable ne prouve que Georget et Géricault se soient fréquentés, ni même connus. De plus, ce n'est que depuis récemment que l'on sait que ni le testament ni l'inventaire après décès du Dr Georget ne font mention de ces portraits d'aliénés. ${ }^{6}$

Depuis la mise en circulation des Monomanes en 1863 , on a cherché à créer des liens, plus ou moins convaincants, entre Géricault et le domaine de la psychiatrie. On a parlé de possible fatigue mentale chez l'artiste, ainsi que d'antécédents familiaux de maladies de l'esprit. Tout cela est sans doute historiquement vérifiable, mais n'éclaire en rien le contexte de production des œuvres. Dans cette même volonté de tirer des conclusions sur le sujet des œuvres, on a aussi soulevé la pratique de Géricault de fréquenter les hôpitaux de sa région pour y obtenir les membres humains qui lui servaient de modèles pour des études indépendantes ou, comme presque tous les commentateurs de Géricault le prétendent, pour en faire des dessins préparatoires pour sa grande réalisation le Radeau de la Méduse. Cette pratique ne s'avère cependant pas un argument recevable en lien avec la réalisation des présumés portraits d'aliénés. L'observation empirique du corps humain ne peut être mise en comparaison avec l'étude des maux de l'esprit, invisibles manifestations de maladie.

Devant un manque de preuves irréfutables qui confirmeraient que les Monomanes de Géricault aient été l'objet d'une commande d'un psychiatre et que les personnes représentées aient été réellement diagnostiquées de cette pathologie, il est alors plus judicieux de ne s'en tenir qu'aux faits concrets. Et ceuxci permettent d'avancer une explication beaucoup plus convaincante sur les liens qui unissent ces œuvres à la psychiatrie naissante: c'est-à-dire que leur désignation à ce titre vient soutenir l'élaboration du mythe de Géricault qui est alors en cours. Cette construction se base sur la mise en relation de deux temporalités et deux sémantiques du terme monomane. Celle des années 1820, qui vise à justifier le projet d'origine de Géricault misant sur l'importance de cette maladie dans l'établissement du prestige de la psychiatrie française à ses débuts, et celle de la seconde moitié du XIX ${ }^{\mathrm{e}}$ siècle, qui réfère au sens nouveau et profane donné au terme monomane, devenu courant dans la population. 
Il est vraisemblable que c'est au moment de leur redécouverte que les Monomanes de Géricault aient reçu leurs titres. Ils leur auraient été conférés par leurs premiers récepteurs connus: Lachèze, Viardot et Blanc. À l'époque où le Dr Lachèze était interne à la Salpêtrière, la monomanie était considérée comme de la première importance dans les milieux psychiatriques. Ceci explique que, quarante plus tard, alors que sa carrière avait pris un tout autre tournant, son souvenir le ramène à cette époque où la monomanie servait à définir la spécialisation médicale émergente. Cependant les Monomanes de Géricault ne correspondent en rien aux descriptions de cas par l'inventeur de la catégorie nosologique, le Dr Jean-Étienne Dominique Esquirol (1772-1840). De plus, en les mettant en relation avec les œuvres commandées à divers artistes ${ }^{7}$ par les premiers psychiatres, il est clair que les Monomanes ne correspondent pas non plus aux codes de représentation de l'aliéné dans les ouvrages scientifiques de l'époque, qui font du malade de l'esprit le contre-modèle de l'image de l'aliéniste sensé et raisonnable. Enfin, la conception de la monomanie comme catégorie scientifique se dissipe graduellement, et le terme en vient à désigner un trouble banal et anodin.

En nommant Monomanes les œuvres découvertes en 1863, les marchands et critiques d'art que sont Viardot et Blanc auraient donc misé sur les deux sens du terme pour en faire des œuvres propres à séduire un acquéreur potentiel. Ainsi, dans la lettre adressée à Charles Blanc, Louis Viardot déclare que les œuvres retrouvées devront sûrement intéresser son interlocuteur ainsi que Tous $^{8}$ les lecteurs de la revue, et ce pour deux raisons: en vertu du nom de leur auteur et par la nature des têtes d'études. Viardot pressent quatre possibles destinations pour ces œuvres qui «peut-être feraient peur aux âmes sensibles, aux complexions nerveuses et délicates» : le cabinet d'un amateur éclairé, un

7. Les seules représentations spécialisées des aliénés depuis la naissance de la psychiatrie sont celles par l'artiste Pierre Maleuvre (1740-1803) dans l'ouvrage de Philippe Pinel (Traité médico-philosophique sur l'aliénation mentale ou la manie, Paris, 1801, réédité 1809 ). Ce sont des œuvres qui demeurent très proches des études encyclopédiques de la période. Par ailleurs, Georges-François-Marie Gabriel a illustré des entrées de dictionnaires pour les articles d'Esquirol en 1814, 1816 et 1818 dans le Dictionnaire des sciences médicales par une société de médecins et de chirurgiens publié chez Panckoucke (Paris, 1812-1822). Certaines de ces œuvres et celles d'autres auteurs non spécifiés ont été gravées par Ambroise Tardieu pour l'ouvrage dont il sera question plus loin: Esquirol, Des maladies mentales considérées sous les rapports médical, hygiénique et médico-légal, Paris, 1838.

8. En majuscules dans le texte d'origine.

9. Viardot, op. cit

10. Ibid.

11. Ibid. établissement comme celui de «l'excellent docteur Blanche», l'amphithéâtre de l'École de médecine, ou encore au Louvre «près du Radeau de la Méduse, du Guide et du Cuirassier». ${ }^{9}$

Ces quelques phrases d'apparence anodine d'un marchand, collectionneur et critique qui cherche à placer des œuvres, orientent l'interprétation de celles-ci. Dépendamment de l'acheteur, le sens que l'on attribue aux œuvres pourrait varier entre deux fonctions: soit médicale et pédagogique, ou pour "ajouter un nouveau lustre au talent de Géricault». ${ }^{10}$ Si elles devaient être acquises par le Dr Blanche, qui était à l'époque le directeur d'une maison de santé privée pour aliénés fortunés, ou achetées pour l'École de Médecine, elles seraient alors interprétées comme des représentations d'aliénés. Si elles s'étaient retrouvées chez un collectionneur amateur de Géricault ou mieux encore, au Louvre, elles auraient alors été perçues comme des têtes d'études, des portraits d'individualités comme Viardot dit en «[avoir] vu plusieurs chez Ary Scheffer et chez d'autres amis du grand artiste». ${ }^{11}$

\section{Les titres des œuvres}

Il est difficile d'associer les cinq portraits de Monomanes à leur désignation de délire spécifique: vol, envie, vol d'enfants, commandement militaire, jeu. En effet, hormis le Monomane du Commandement militaire | fig. 1 | que l'on peut rapprocher d'un délire militaire, grâce à son képi, à sa médaille, et à son costume, 

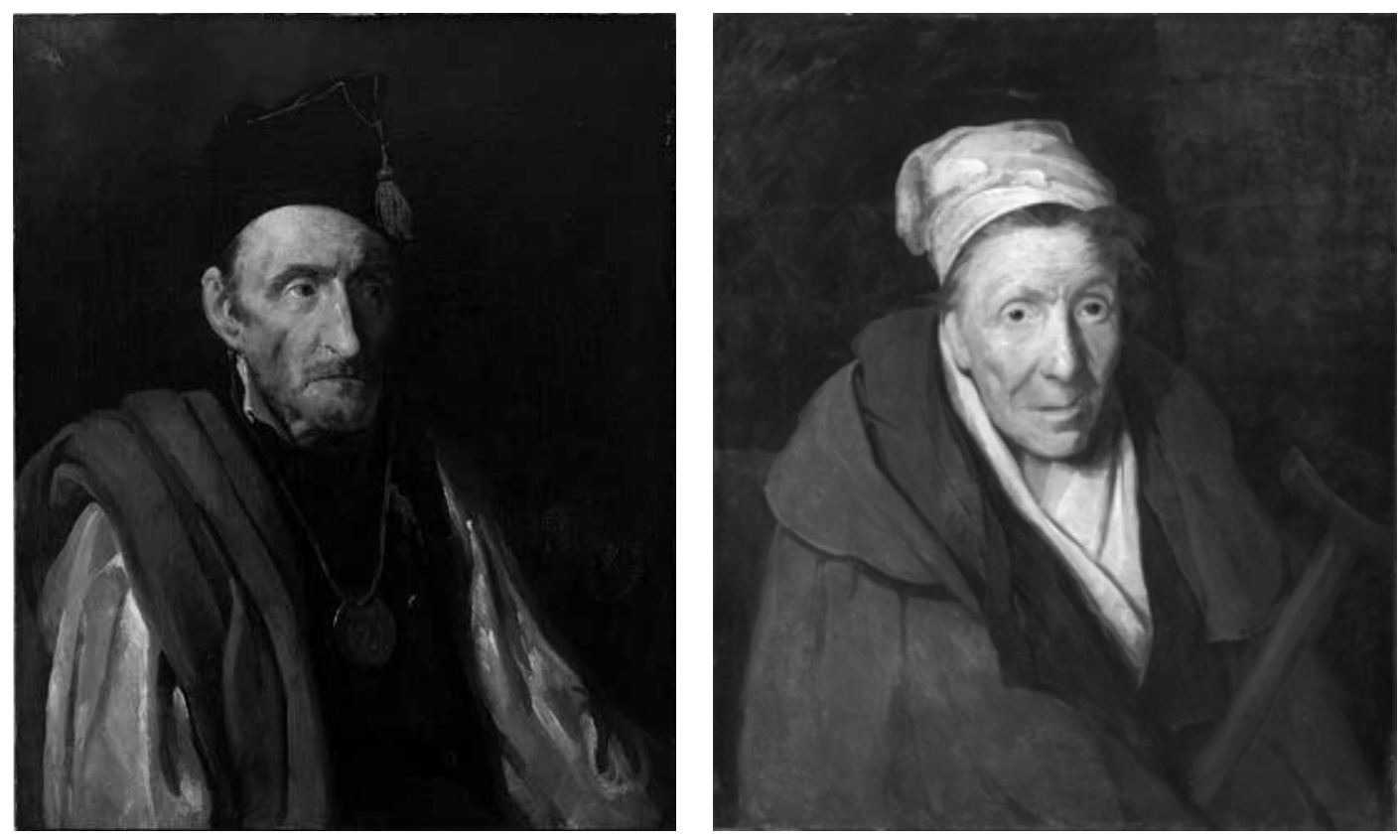

les autres descriptions de Viardot ne nous permettent pas de discerner les troubles spécifiques que celui-ci lit dans l'apparence des sujets représentés. Comment la figure de la vieille dame avec une béquille nous parle-t-elle du délire du jeu qui l'anime? | fig. 2 | Rien dans son expression perdue, presque hagarde, ne nous permet de supposer qu'elle est «absorbée dans des calculs d'ambes, ternes et quaternes». ${ }^{12}$ Comment le monomane désigné comme un voleur peut-il être compris comme tel? Son regard est doux et c'est pourtant sur ses traits, qu'il qualifie d'assez beaux, que Viardot lit cette maladie, évoquant un regard équivoque et une bouche de coquin qui fait un mauvais coup. Le monomane qualifié du vol des enfants se reconnaît, toujours selon Viardot, par une figure paterne, un front bombé, un œil doux, une bouche engageante. Enfin, la Monomane de l'envie, cette femme qu'il dit avoir porté, en son temps, le surnom de la Hyène de la Salpêtrière, se reconnaît par des grincements de la bouche et des injections de sang dans les yeux qui expriment selon lui, on ne sait pourquoi, non la rage, non la fureur, non la méchanceté, mais l'envie. En les qualifiant de monomanes, Viardot et Lachèze font se rencontrer les deux niveaux sémantiques du terme: catégorie nosologique de la science aliéniste dans les années 1820 , telles que dans le souvenir du Dr Lachèze et expression populaire devenue présente dans le langage courant du Second Empire. S'expliquent alors les incohérences des descriptions des tableaux dans la lettre de Viardot par rapport à la définition médicale de la monomanie, soulevant un doute sérieux sur l'état des personnes représentées et encore davantage sur leur délire spécifique.

Géricault ne signait pas ses œuvres, ne les datait pas. C'était une pratique courante au XIX ${ }^{\mathrm{e}}$ siècle. De plus, les œuvres étaient, très souvent, nommées par les marchands et les critiques. Dans le corpus de Géricault, les seuls titres qui 
Figure 1. (Ci-contre, à gauche) Théodore Géricault, Monomane du commandement militaire, vers 1820 , huile sur toile, $81 \times 65 \mathrm{~cm}$. Collection Oskar Reinhart «Am Römerholz», Winterthur.

Figure 2. (Ci-contre, à droite) Théodore Géricault, Monomane du jeu, vers 1820 , huile sur toile, $77 \times 64,5 \mathrm{~cm}$. Paris, musée du Louvre. (C RMN-Grand Palais/Art Resource, NY, photo: Philippe Fuzeau.
13. Gladys Swain et Marcel Gauchet, Dialogue avec l'insensé: essais d'histoire de la psychiatrie, Paris, 1994, p. XXXIII.

14. Le mot signifie étymologiquement bile noire et renvoie encore à la théorie humorale d'Hippocrate.

15. Olivier Eymann, Être fou au $\mathrm{xIX}$ siècle: moralisation et normalisation des internés d'office d'un asile de province, Paris, 2006, p. 103-106. On peut aussi référer aux textes originaux pour une documentation plus détaillée: Pinel, Traité médico-philosophique, op. cit.; Esquirol, Des maladies mentales, op. cit. pourraient provenir de lui, ou qui à tout le moins ont été donnés de son vivant, sont clairement descriptifs du sujet représenté. Ce sont ceux qui nous sont connus par les livrets des Salons: Portrait de M.D. (1812), rebaptisé Officier de Chasseurs à cheval de la garde impériale chargeant; Cuirassier blessé (1814); Scène de naufrage (1819), rebaptisée Radeau de la Méduse. Ou encore les vignettes de lithographies: Boxeurs, Maréchal-ferrant, Chevaux allant à la foire, Factionnaire suisse au Louvre. C'est donc en 1863 que les œuvres qui nous intéressent ont été nommées Monomanes par Viardot se basant sur les souvenirs du Dr Lachèze. Pour comprendre pourquoi ce titre a pu leur être attribué, il faut chercher les premiers indices dans les années 1820, années de leur création, de définition de la nouvelle catégorie de maladie mentale, et de la présence active du Dr Lachèze à la Salpêtrière.

\section{La monomanie en 1820}

Avec la naissance de la nouvelle spécialisation médicale qu'est l'aliénisme, ancêtre de la psychiatrie, le fou de l'Ancien Régime en France devient, au début du XIX ${ }^{\mathrm{e}}$ siècle, l'aliéné. La différence est importante. Contrairement à la pratique inhumaine qui fait du malade de l'esprit un être incurable, enfermé et traité à la limite des conditions d'humanité, l'insensé devient alors l'objet d'étude d'une communauté spécialisée de médecins qui s'engagent à le comprendre et à le guérir. Le moment fondateur de la psychiatrie repose sur la nouvelle prise en cause du sujet chez le fou, alors que Philippe Pinel (17451826), considéré aujourd'hui comme le père de la psychiatrie française, inaugure la notion de traitement moral. Cette nouvelle volonté de traitement des malades mentaux s'adresse à la partie sensée qui demeure toujours présente dans l'esprit du patient, considérant l'aliéné mental comme un être à réincorporer dans une société normalisée. Le principal disciple de Pinel, Jean-Étienne Dominique Esquirol est vu comme celui qui a donné le plus grand essor de professionnalisation à l'aliénisme, peut-être même «le véritable fondateur (en pratique) de l'aliénisme». ${ }^{13}$ Pour comprendre cette assertion, il faut connaître les nombreux accomplissements d'Esquirol, qui a poursuivi l'œuvre de Pinel et qui a permis à l'aliénisme de s'établir et de devenir une science médicale autonome et puissante.

Esquirol est en effet reconnu pour avoir complété le système de classification des maladies mentales établi par Pinel et pour avoir basé ses classifications sur des observations cliniques. Les classifications qu'il établit se fondent sur les quatre catégories de maladies mentales décrites par Pinel dans son Traité médico-psychologique de l'aliénation mentale de 1801 , soit 1) la mélancolie; ${ }^{14}$ 2) la manie, presque synonyme de folie au XIX ${ }^{e}$ siècle, un secteur nosographique très large et qui concerne à l'époque, près de la moitié des malades; 3) la démence, un affaiblissement progressif des fonctions intellectuelles; 4) l'idiotisme ou l'imbécillité, une abolition totale des fonctions de l'entendement, un arrêt de développement intellectuel complet en rapport avec un arrêt de développement physique. ${ }^{15}$ Esquirol ajuste le système de classification de Pinel en cinq catégories nosologiques. Il conserve manie, démence et idiotisme, et change le nom de la mélancolie pour lypémanie. Enfin, il crée la catégorie de la monomanie, qui se distingue de l'insanité générale par son caractère de délire partiel et qui a la particularité d'absorber momentanément toutes les facultés de l'intelligence, alors que par ailleurs, le sujet atteint de ce 
16. Esquirol, «Monomanie», dans Dictionnaire des sciences médicales Paris, 1819, p. 115

17. Je conserve l'orthographe originale des citations des textes d'époque.

18. Esquirol, «Monomanie», op. cit., p. 115-116.

19. Étienne-Jean Georget, Examen médical des procès criminels des nommés Léger, Feldtmann, Lecouffe, Jean-Pierreet Papavoine, dans lesquels l'aliénation mentale a été alléguée comme moyen de défense, suivi de quelques considérations médico-légales sur la liberté morale, Paris, 1825; Discussion médico-légale sur la folie ou aliénation mentale, suivie de l'examen du procès crimine d'Henriette Cornier, et de plusieurs autres procès dans lesquels cette maladie a été alléguée comme moyen de défense, Paris, 1826; Des maladies mentales, considérées dans leurs rapports avec la législation civile et criminelle, Paris, 1827; et Nouvelle discussion médico-légale sur la folie ou aliénation mentale, suivie del'examen de plusieurs procès criminels dans lesquels cette maladie a été alléguée comme moyen de défense, Paris, 1828.

20. Ginette Jubinville, «Représenter et construire la psychiatrie en France, 1801-1863. L'art des premiers aliénistes», Thèse de doctorat, Université de Montréal, 2014.

21. Ceci permet de plus de couper à la source le possible argument qui pourrait surgir, à savoir que les Monomanes auraient pu être peints dans un moment où ils ne sont pas en crise de leur délire spécifique. type de folie agit de manière conforme à la norme-un concept qu'il identifie comme folie raisonnante:

Nous conservons le nom de monomanie au délire partiel, dépendant de passions excitantes, expansives et gaies. La monomanie est une espèce intermédiaire entre la lypémanie et la manie; elle participe de la lypémanie (mélancolie) par la fixité et la concentration des idées et de la manie par l'exaltation des idées et par l'activité physique et morale. ${ }^{16}$

Ainsi, dès la création de la catégorie nosologique, Esquirol décrit les malades atteints de monomanie comme des personnes agitées et joyeuses: «Les monomaniaques sont gais, vifs, pétulans, ${ }^{17}$ audacieux, téméraires [...] bruyants, bavards [...] heureux, contents et joyeux, rient, chantent, dansent, se trouvent au comble du bonheur et de la félicité. ${ }^{18}$ Pourtant, il est impossible de trouver sur les expressions et les faciès des personnes représentées par Géricault quelque signe qui corresponde de près ou de loin à cette description.

Dans les années 1820 , la monomanie est essentielle à la reconnaissance de la nouvelle spécialisation médicale, malgré certaines dissensions au sein de la science aliéniste. Par son caractère partiel, le concept de monomanie est alors l'instrument idéal pour faire comprendre à la population que le regard de l'aliéniste sur le malade mental est le seul apte à déceler sa maladie. Si le profane peut aisément identifier la folie, la monomanie ne peut être révélée que par l'œil averti de l'aliéniste.

De plus, au plan professionnel, l'aliéniste aspire à la même reconnaissance, au même degré de dignité et de respectabilité que celui conféré aux membres de la profession juridique. Une querelle de frontières s'amorce alors rapidement, affirmant la compétence unique des aliénistes à identifier la folie dans les procès criminels. Le disciple favori d'Esquirol, le Dr Étienne-Jean Georget produit d'ailleurs entre 1825 et 1828 des publications qui ont un grand retentissement sur les rapports entre médecine et loi, instaurant en France la psychiatrie médico-légale. ${ }^{19}$ Ceci peut expliquer le fait qu'en 1863 le Dr Lachèze, absent de France depuis quarante ans et loin de l'évolution de ces discussions, ait confié à Viardot des souvenirs du temps où la monomanie était primordiale dans le développement de l'aliénisme et que dans son souvenir la monomanie et la figure de Georget soient liées de manière indéfectible.

\section{Les Monomanes face aux codes de représentation scientifique des aliénés}

Un argument supplémentaire permet de mettre en doute la condition de monomanes des personnes représentées dans les œuvres de Géricault. Mes recherches sur l'art commandé par les premiers aliénistes ont démontré que les représentations d'aliénés, lorsqu'elles sont faites pour des traités psychiatriques ou pour des projets de catégorisation, respectent un certain code de représentation qui les pose comme les contre-modèles des représentations des aliénistes. ${ }^{20}$ Les Monomanes de Géricault ne respectent en rien cette codification. ${ }^{21}$ En effet, pour appliquer les nouveaux principes qui s'opposent aux traitements physiques, visant à extirper la folie du corps préconisés jusqu'alors, le traitement moral repose largement sur la personne même de l'aliéniste qui impose sa présence physique, son corps, comme modèle d'ordre, de raison et d'autorité à l'esprit dérangé de ses patients. Comme Sander L. Gilman a su le mettre en lumière, l'altérité et les stéréotypes sont construits par l'image dans une culture donnée alors que chaque catégorie est 
Figure 3. Pierre-Auguste Pichon, Jean-Étienne Dominique Esquirol, s.d., huile sur toile, $128 \times 96 \mathrm{~cm}$. (c) Bibliothèque de l'Académie nationale de médecine (Paris), photo: Philippe Fuzeau.
22. Ma traduction de: «Each category is perceived as either the embodiment or the antithesis of the group which has provided the category». Sander L. Gilman, Seeing the insane, New York et Toronto, 1982 , p. XI.

23. Elles ont été acquises par la Bibliothèque nationale de France directement de l'artiste en 1831 et en 1836 où elles sont toujours conservées.

24. Élève d'Ingres, Pichon a exposé au Salon à partir de 1835 et a reçu une médaille de bronze en 1843 , une d'argent en 1844 , et une d'or en 1846 (Bénézit 2006). II se spécialise dans la peinture religieuse et dans le genre du portrait.

25. Esquirol, op. cit.

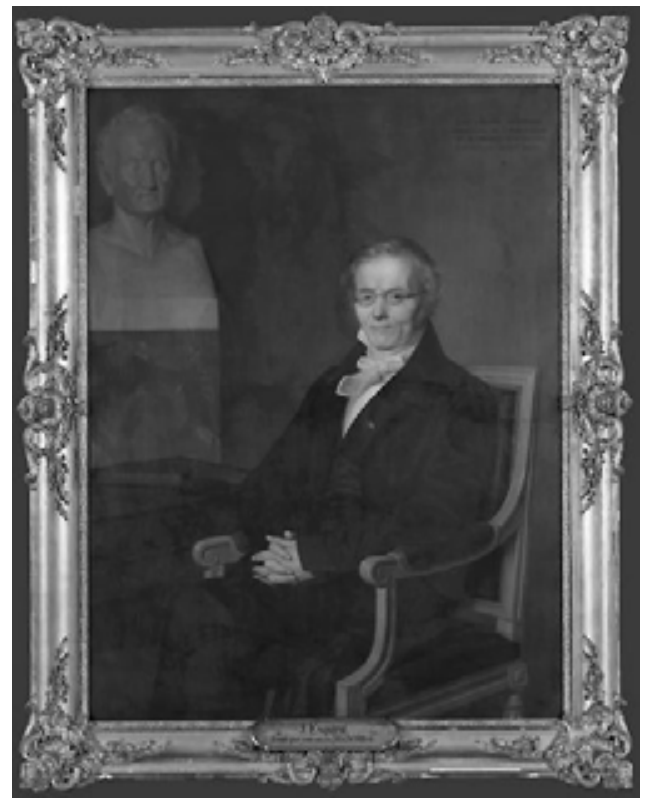

vue soit comme l'incarnation soit comme l'antithèse du groupe qui a produit la catégorie. ${ }^{22}$ Ainsi, dans les représentations de malades dessinés pour Esquirol, les seules représentations d'aliénés desquelles il est possible de rapprocher les Monomanes de Géricault sont celles du Recueil de Têtes d'Aliénés dessinées par Georges-François-Marie Gabriel pour un ouvrage de M. Esquirol, relatifà l'aliénation mentale. Entre 1813 et 1823 , Georges-François-Marie Gabriel (1775-1836) produit une série de dessins de malades qui sont particulièrement sensibles et qui savent reconnaître la subjectivité du patient. Ces œuvres les présentent avec leurs spécificités, leurs différences, sans engager la notion d'altérité et de mise à l'écart. ${ }^{23}$ Elles n'ont pourtant pas été retenues par l'aliéniste qui les a commandées et elles sont demeurées en possession de l'artiste. Les représentations d'aliénés qui ont retenu l'attention d'Esquirol sont celles qui placent le patient en position d'observé, à l'opposé du médecin observateur, construisant ainsi le contre-modèle de l'aliéniste. Comparons le Portrait d'Esquirol par Pierre-Auguste Pichon (1805-1900), ${ }^{24}$ et les représentations de malades dans le traité psychiatrique de la même période, Des maladies mentales considérées sous les rapports médical, hygiénique et médico-légal, illustré pour Esquirol de vingt-sept lithographies d'Ambroise Tardieu (1788-1841). ${ }^{25}$

Le portrait d'Esquirol | fig. 3 | peut être vu aujourd'hui comme le prototype de l'image du psychiatre. Esquirol y est représenté assis dans un fauteuil, à l'écoute de son patient, les épaules et les bras au repos, détendu, attentif, l'expression de son visage bienveillante. Sa physionomie est celle d'une personne sereine et en contrôle d'elle-même. Il porte de petites lunettes ovales cerclées de métal. Sa coiffure bouclée avec une raie sur le côté et sa petite mèche sur le front indiquent un homme qui soigne son apparence. Le visage est dessiné par les traits marqués d'un sourire un peu pincé avec une très légère fossette sur la joue. Les sourcils, qui servent à encadrer l'expression 
26. Le buste de Philippe Pinel en marbre blanc est l'œuvre de Pierre-Alfred Robinet et date de $1856(60 \times 36 \mathrm{~cm})$. Il est conservé à l'Académie nationale de médecine de Paris. Ce serait une reprise en marbre d'un plâtre réalisé en 1827 par Théophile Bra. La difficulté de datation du portrait de Pichon rend impossible de savoir de quelle version du buste il s'agit.

27. Esquirol, op. cit., planches xx et xxII.

28. Dans l'historique de la représentation des aliénés pour les premiers psychiatres, les premières œuvres s'approchent de portraits, cadrés en buste. Ce sont les dessins de Georges-François-Marie Gabriel dont nous avons parlé plus haut. Mais dès le traité de 1838 à l'étude ici, il a fallu réincorporer le corps dans la représentation pour mieux circonscrire les signes de démence et illustrer le propos de l'aliéniste. C'est à la fois une négation de la croyance en la physiognomonie et une nouvelle stigmatisation de l'aliéné. Pour d'autres traités spécialisés de l'époque, consulter: Bénédict-Augustin Morel, TraitéThéorique et pratique des maladies mentales, Paris, 1852, ou du même auteur, Traitédes dégénérescences physiques, intellectuelles et morales de l'espèce humaine et des causes qui produisent ces variétés maladives, Paris, 1857.

29. Je renvoie à lan Goldstein, dont l'analyse des enjeux sur la monomanie nous est très éclairante. Jan Ellen Goldstein, Consoler et classifier: l'essor de la psychiatrie française, Le Plessis-Robinson, 1997, chapitre 5, p. 209-265. forte du regard pénétrant, présentent un dessin sinueux; les arcades sourcilières se rejoignent sur le front en une ligne courbe. Il porte un vêtement bourgeois avec veste ouverte, gilet boutonné, chemise, faux-col, cravate et la rosette d'une décoration est visible, bien rouge. L'image du psychiatre à l'écoute est construite dans ce portrait; sa légitimité professionnelle est aussi affirmée puisque Esquirol se fait représenter en présence du buste de son maître Philippe Pinel. ${ }^{26}$ La composition de l'œuvre construit ainsi une hiérarchie de rôles entre le maître et le disciple d'une part, mais surtout entre l'aliéniste et le malade dont la présence est implicite sous le regard d'Esquirol. En effet, le regard de l'aliéniste sur le malade est à la base du rapport d'autorité paternaliste entre psychiatre et patient.

La rhétorique des œuvres lithographiées par Tardieu illustrant le traité psychiatrique d'Esquirol, Des maladies mentales considérées sous les rapports médical, hygiénique et médico-légal, est à l'opposé de celle de Pichon. En effet, dans les illustrations de ce traité, l'aliéné est exposé à notre regard. | fig. 4 | Le malade est vu en plongée. L'espace, ainsi construit autour de lui, le place en situation de vulnérabilité, incapable d'échapper au regard et à l'observation, la ligne d'horizon très basse induisant une vision invasive. De surcroît, un travail faussé de la perspective, dans les planches où des éléments s'y prêtent, contribue à déstabiliser malade et spectateur. Il implique un glissement du malade hors de lui-même et révèle sa condition précaire. | fig. 5 |

L'habillement est une autre caractéristique qui distingue les aliénés de l'ouvrage d'Esquirol. Ils sont, pour la plupart, vêtus de tenues d'hôpital, alors que tous les médecins sont portraiturés en habits civils, officiels, bourgeois. Mais il y a plus: la pâleur généralisée des vêtements des aliénés nous porte à croire qu'ils sont blancs, et ce, même, contre toute probabilité, dans les quelques cas où les malades portent des habits civils. ${ }^{27} \mid$ fig. 6 |

Outre ses vêtements, tout l'environnement de l'aliéné est blanc: pavés, sol, murs, banquettes, chaises, et un effet de lumière frontale et crue, avec un minimum d'ombre portée, contribue à renforcer cette impression d'univers vide et exposé. De ce fort contraste induit entre les visages qui sont sombres et ombrés et le blanc environnant, l'attention se trouve portée sur la physionomie du malade. Son teint apparaît particulièrement altéré sous l'effet des passions qui l'animent, exprimant ses troubles émotifs.

On le constate, les portraits produits par Géricault dans les années 1820 ne répondent en rien à la codification iconographique du malade mental, que ce soit dans l'apparence, l'expression, la gestuelle, l'environnement, les attributs, le cadrage en buste, l'angle de vision. ${ }^{28}$ Ce constat des liens improbables entre les œuvres de Géricault et le domaine de la psychiatrie du début du siècle, nous renvoie maintenant à leur contexte de réception en 1863, quarante ans après leur création, moment où ils reçoivent leur désignation de monomanes.

\section{La monomanie en 1863}

Si dans les années 1820 , alors que la psychiatrie naissante cherchait à faire reconnaître son champ de spécialisation, l'identification de cette maladie mentale était prépondérante, dans les années 1860 il en va tout autrement. La catégorie nosologique était devenue obsolète. ${ }^{29}$ Elle vit un déclin dès les années 1830-1840, mais le véritable tournant se situe en 1854 lorsque Jean- 


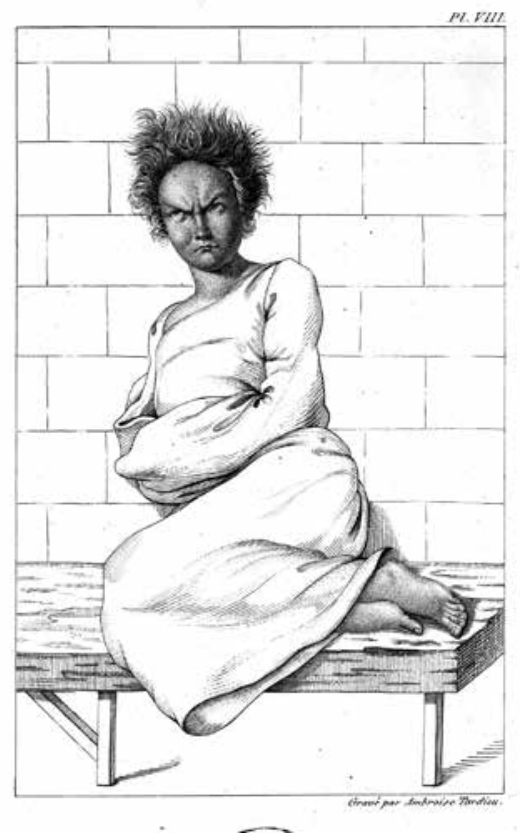

Figure 4. Ambroise Tardieu,

1838, Maniaque, Planche VIII,

lithographie, tirée de l'ouvrage:

Étienne Esquirol, Des maladies

mentales considérées sous les

rapports médical, hygiénique et

médico-légal, Paris, 1838.

Photo: BIU Santé. www.biusante.

parisdescartes. fr/histmed/

image?02529

Figure 5 (en bas, à gauche).

Ambroise Tardieu, 1838,

Maniaque, après la guérison,

Planche Ix, lithographie, tirée

de l'ouvrage: Étienne Esquirol,

Des maladies mentales considérées

sous les rapports médical, hygiénique

et médico-légal, Paris, 1838.

Photo: BIU Santé. www. biusante.

parisdescartes.fr/histmed/

image?01360

Figure 6 (en bas, à droite).

Ambroise Tardieu, 1838, Idiot,

Planche XxII, lithographie, tirée

de l'ouvrage: Étienne Esquirol,

Des maladies mentales considérées

sous les rapports médical, hygiénique et médico-légal, Paris, 1838.

Photo: BIU Santé. www. biusante. parisdescartes.fr/histmed/ image?02527

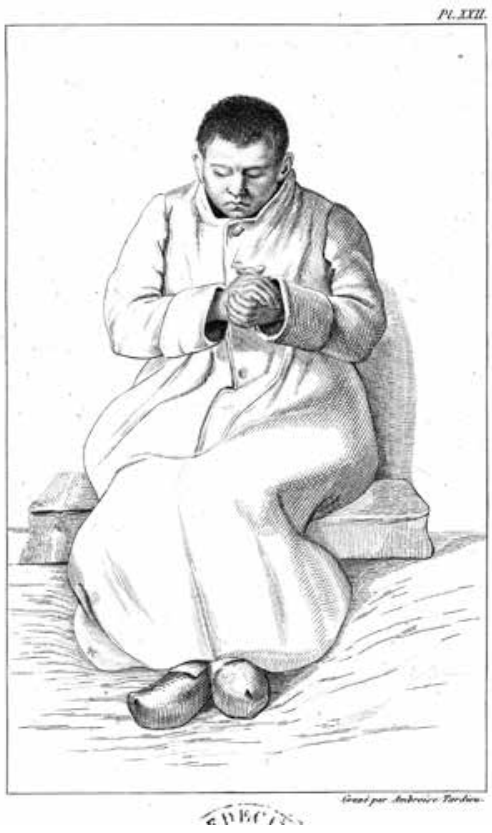


30. Jean-Pierre Falret, Dela non-existence de la monomanie, $\mathrm{Pa}$ ris, 1854 .

31. Le chapitre 7 de Goldstein, Consoler et classifier (op. cit.), porte sur ces deux camps de la pensée et de la théorie aliénistes à ses débuts. p. 307-351.

32. Goldstein, op. cit., p. 258.

33. Dictionnaire des sciences médicales, op. cit; Pierre-Hubert Nysten, Dictionnaire de médecine, de chirurgie, de pharmacie, des sciences accessoires et de l'art vétérinaire: ouvrage augmentéde la synonymie grecque, latine, allemande, anglaise, espagnole et italienne et suivi d'un glossaire de ces diverses langues, $\mathrm{Pa}$ ris, Londres, New York, 1855; FélixAndré-Augustin Poujol, Dictionnaire de médecine-pratique et des sciences qui lui servent de fondements, Paris, 1857; Dictionnaire de la Langue Française E. Littré, Paris, 1874, Tome 3 (I-P); Dictionnaire Larousse: www. larousse.fr/dictionnaires/francais/ monomanie/52346.

34. Goldstein, op. cit., p. 211.

35. Je rappelle que l'Hospice de Charenton est au XIX ${ }^{\mathrm{e}}$ siècle un asile parisien célèbre.
Pierre Falret, pourtant un membre du cercle d'Esquirol, rédige une thèse de médecine intitulée «De la non-existence de la monomanie». ${ }^{30}$ Durant ces années, au cœur même de la science aliéniste, la catégorie nosologique est remise en question par un groupe de psychiatres, réfutant sa définition même qui stipule qu'une partie de l'esprit serait en délire sur un point spécifique alors que le reste de la raison resterait intouché. L'aliénisme vit alors une crise de légitimité, tant face à la médecine générale, au sujet de la multiplicité des maladies mentales, qu'en son sein même. La discipline se divise alors en deux camps: ${ }^{31}$ celui qui définit la maladie mentale dans sa dimension physiologique et celui qui la définit dans sa dimension psychologique. Cette tension, qui est présente depuis le début de l'aliénisme, se condense alors autour de la catégorie nosologique qu'est la monomanie, particulièrement pour la large intrusion qu'elle permet du monde aliéniste dans le monde juridique.

Durant ce processus, le sens du terme monomanie passe dans le langage populaire. Il est désormais utilisé pour exprimer les excès et les bizarreries de comportement des citoyens, alors que la science psychiatrique ne la diagnostique pratiquement plus. Le déclin des cas confirmés de monomanie dans les hôpitaux est révélé par Jan Goldstein qui indique que, d'après ses recherches dans les registres d'admissions des hôpitaux, sur l'ensemble des malades, 10\% de cas sont répertoriés en 1848 , qu'il n'y en a plus que $8 \%$ en 1860 , et $2 \%$ en 1865. La catégorie disparaît tout à fait en $1870 .^{32}$

Dans les dictionnaires, le même déclin d'intérêt pour la maladie s'observe. Dans le dictionnaire médical Panckoucke, par exemple, où le terme paraît pour la première fois en 1819, l'article signé par Esquirol est long de douze pages. En 1855 le Dictionnaire médical Nystens n'y consacre que vingt lignes. Le Poujol de la même année y dédie six lignes. Dans les dictionnaires généraux, si le Littré de 1874 définit encore la monomanie comme un terme médical: «Folie ou délire sur un seul objet», le Larousse d'aujourd'hui n'y consacre que quelques mots particulièrement vagues: «toute affectation psychique qui n'affecte que partiellement l'esprit». ${ }^{33}$ La monomanie est donc passée de préoccupation scientifique à une "catégorie culturelle générale» ${ }^{34}$ définissant les citoyens atteints d'obsessions somme toute bénignes. Ainsi, les raisons qui ont occasionné son déclin dans le domaine médical sont celles-là mêmes qui ont donné naissance à son succès populaire. Dans la seconde moitié du XIX siècle, le terme peut s'appliquer à n'importe qui. Toute douce folie devient monomanie et toute petite obsession fait le ou la monomane. La catégorie nosologique devient comportement populaire et répandu dans la société.

Le passage du sens psychiatrique de la monomanie à son sens populaire se fait graduellement, et en partie dans la presse où les images et les articles de vulgarisation se croisent. On en voit un exemple dans les caricatures créées par Honoré Daumier. Déjà en 1832, dans la revue La Caricature, Daumier avait transposé le concept de la monomanie hors des murs de l'asile. Mais la référence à la folie était encore très claire, faisant figurer les membres du gouvernement de Louis-Philippe comme des aliénés réunis dans ce qu'il appelle le Charenton Ministériel. ${ }^{35}$ | fig. $7 \mid$ Dans cette image, tous les personnages gesticulent, se contorsionnent, agissent de manière insensée, portent des coiffes ridicules, des vêtements extravagants. La rupture de la monomanie avec le domaine de la santé mentale n'est pas encore complètement établie et la 


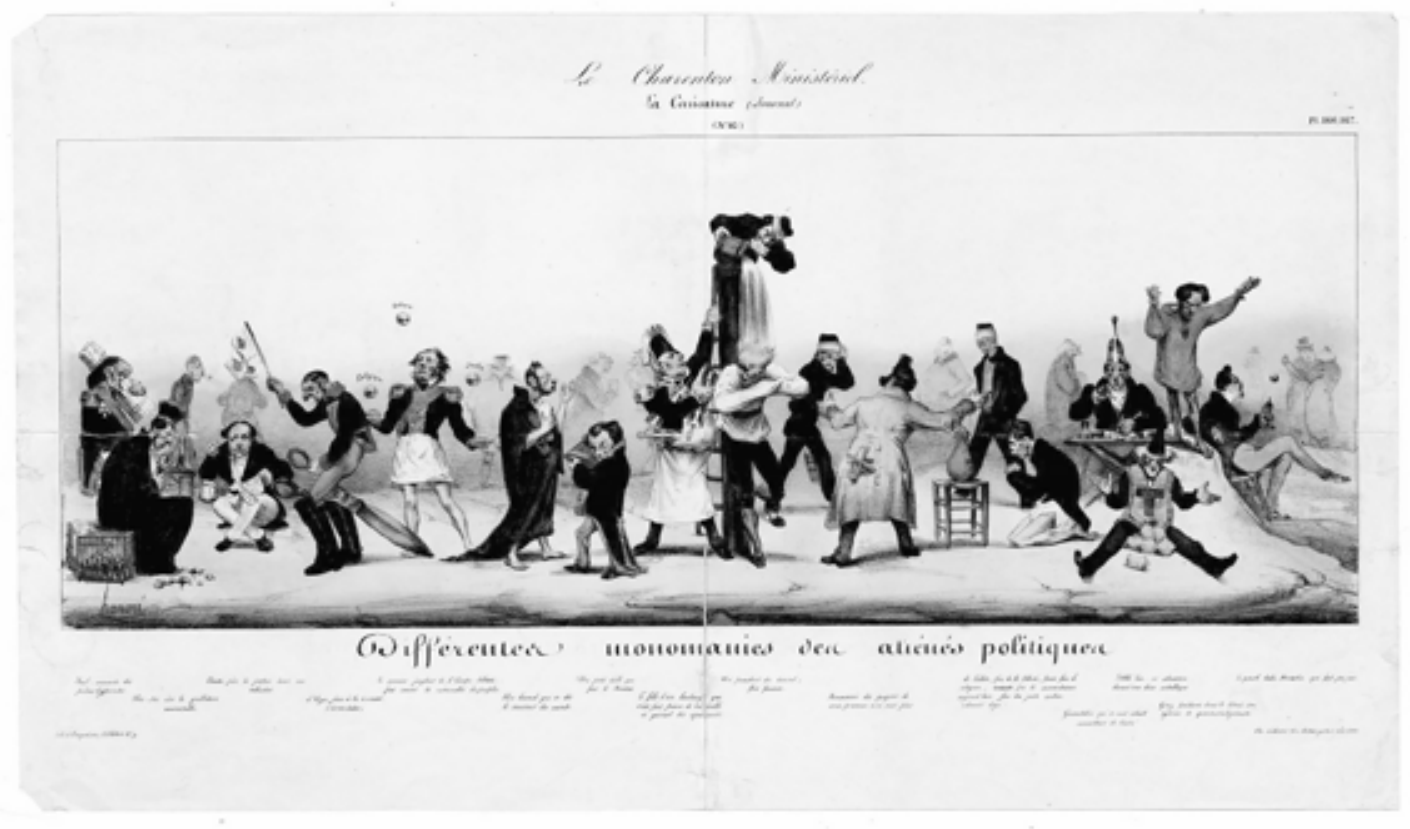

Figure 7. Honoré Daumier,

Le Charenton Ministériel, Différentes monomanies des aliénés politiques, lithographie, $19,3 \times 50,7 \mathrm{~cm}$,

publié dans La Caricature, 31 mai

1832, Photo: (c) Trustees of the British Museum.

Figure 8. Honoré Daumier, Le Régulateur, série Monomanes, lithographie, $37 \times 25,3 \mathrm{~cm}$, publié dans Le Charivari, 5 novembre 1840. Photo: () Trustees of the British Museum.

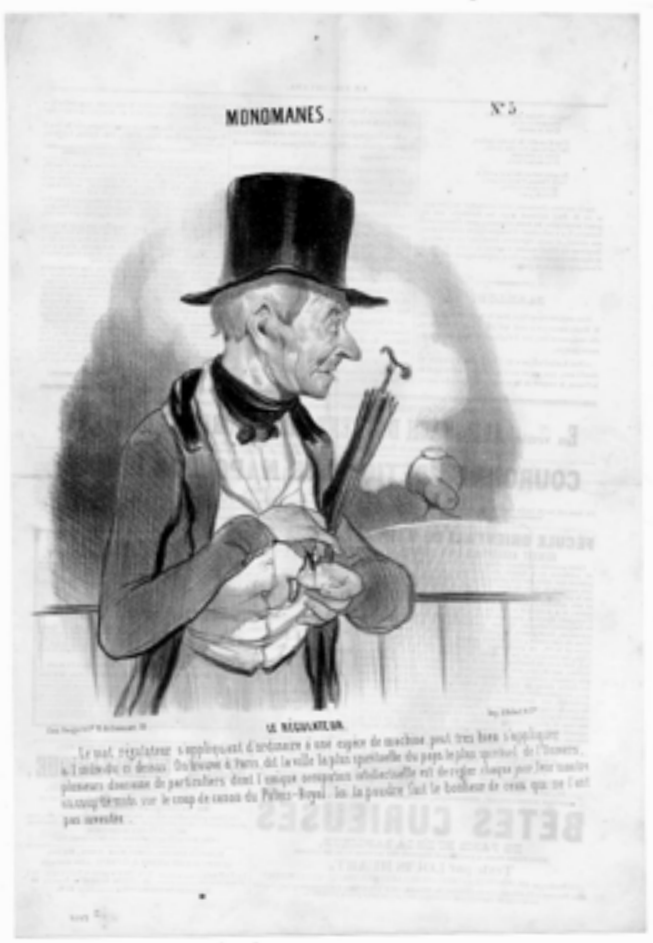




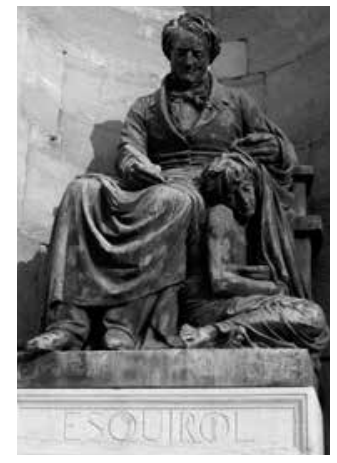

Figure 9. Armand Toussaint, Esquirol, 1861, bronze et fonte, 180 (h) $\times 158$ (la) $\times 107(\mathrm{pr}) \mathrm{cm}$, inscription en bas à gauche: Fdrie de Eck et Durand, Saint-Maurice, Hôpital Esquirol. Photographie de l'auteure (2012).
36. Sander L. Gilman a passé en revue ces motifs dans l'iconographie occidentale du Moyen-âge au XIXe siècle. II a établi quatre catégories visuelles pour rendre visible la folie et marquer l'altérité du fou: 1) l'apparence faciale, 2) la position étrange du corps, contorsionné, 3) tête et membres agités, le geste désordonné et incohérent, 4) l'expression exagérée, signe d'un état psychologique trouble et envahissant. D'autres marqueurs visuels, recensés par Gilman, sont le bâton du fou, et bien sûr, l'environnement dans lequel le fou est représenté, l'isolant de la réalité. J'ajouterai à ces catégories, le collier à clochettes du fou, le chapeau à cornes ou tout couvre-chef extravagant et souvent le costume de bouffon. Voir à ce sujet: Gilman, op. cit.

37. Ces œuvres sont consultables sur le site suivant: www. daumier-register.org/werklist.php?x_SerielD=158\&z_Seriel $\mathrm{D}=\% 3 \mathrm{D} \% 2 \mathrm{C} \% 2 \mathrm{C}$

38. Voir note 19 , les procès de Léger, Feldtmann, Lecouffe, Jean-Pierre, Papavoine, Henriette Cornier.

39. Charles Blanc, Histoire des Peintres de toutes les Écoles. École française, Paris, 1861, p. 1.

40. Ibid., p. 1.

41. Ibid., p. 11. satire de Daumier consiste justement à appliquer aux personnages politiques les comportements réservés aux fous dans la tradition de représentations. ${ }^{36}$

En 1840-1841, le Charivari publie une série de huit caricatures de Daumier intitulée Monomanes. ${ }^{37}$ Avec ces œuvres, le sens du terme a poursuivi sa transformation et la connotation à la folie est plus douce, les personnages, outre leurs habitudes particulières, ne sont pas affligés de comportements pathologiques. Daumier y représente des personnalités banales et humoristiques, qui sont loin des cas de monomanie homicide des procès des années 1820. ${ }^{38}$ De profil ou de face, leurs regards n'interpellent jamais le spectateur, signe indubitable d'intériorisation de la douce obsession que leur esprit leur impose. À la ville, à la campagne ou dans l'intimité de leur foyer, ils s'adonnent à des activités fort diverses. Le Bêtophile est occupé à prendre soin de sa petite ménagerie personnelle, souriant béatement à un oiseau en cage. Le Savant qui ne sait que «des choses niaises et inutiles», semble plongé dans ses réflexions creuses, le Brodeur est bien concentré sur son travail et le Guittariste-amateur [sic] a l'air inspiré, les yeux au ciel. Le Régulateur | fig. $8 \mid$ règle obsessivement sa montre chaque jour sur le coup de midi. Le Chasseur parisien dont la chasse n'est que prétexte à une ballade dans la nature, se promène le fusil désamorcé sous le bras, plongé dans sa lecture. Le Malade imaginaire, l'air inquiet, prend son propre pouls de manière compulsive et enfin l'Amateur de café est un homme à l'aspect débraillé d'un manant qui ne peut s'offrir que de la chicorée. Les œuvres de Daumier publiées dans la Caricature et le Charivari ont connu une large diffusion et ont très certainement contribué à faire du terme de monomanie un concept applicable en dehors de l'asile.

Quand les œuvres de Géricault sont redécouvertes en 1863, la situation de la science aliéniste n'est donc plus la même. À cette période déjà, la jeune psychiatrie peut se reposer sur une reconnaissance institutionnelle. La loi de 1838, dite Loi des Aliénés, qui donne beaucoup de pouvoir et de légitimité aux aliénistes assure une professionnalisation de leur discipline. La reconnaissance officielle de son institutionnalisation se fait en 1852, par la création de la Société médico-psychologique. La monomanie y est remise en question et les prises de position divergentes de ses membres peuvent se déployer au grand jour: la science psychiatrique n'en dépend plus pour sa légitimation.

\section{La construction du mythe}

En 1863, les circonstances propices à la construction du mythe de l'artiste convergent. Parmi les auteurs repris par Charles Clément pour sa biographie de 1867 , Charles Blanc lui-même a écrit en 1861 sa propre glorification de l'artiste, où il en fait un des héros de l'École française, celui qui "passe pour avoir donné en peinture le signal de la réforme». ${ }^{39}$ Il n'est pas étonnant que celui qui voit chez Géricault l'artiste qui «poursuit la réalité plus loin encore [que Gros] en y découvrant une poésie profonde», ${ }^{40}$ ait pressenti ces cinq portraits comme des œuvres importantes, découvertes alors qu'une certaine fièvre entourait tout ce que l'artiste avait produit. Charles Blanc reconnaît en ces œuvres la recherche de Géricault «d'ennoblir l'humanité, de prêter de la poésie à son histoire et de nous intéresser à ses malheurs». ${ }^{41}$

Le 21 novembre 1862, l'inauguration de la Statue d'Esquirol, | fig. 9 | réalisée par Armand Toussaint, à la Maison Impériale de Charenton, est un prétexte 
42. L'Empire prend alors une tournure plus libérale. Après l'amnistie générale décrétée au retour de la Campagne d'Italie, Napoléon III recherche de nouvelles victoires dans l'administration de son pays.

43. Maison impériale de Charenton, Inauguration de la statue d'Esquirol, «Discours de Parchappe», Paris, 22 novembre 1862 , p. 10

44. Les détails de leur destinée sont notés avec précision par Germain Bazin dans son catalogue raisonné de 1994: Germain Bazin, Théodore Géricault: étude critique, documents et catalogue raisonné, Paris, $8 \mathrm{v}$. 1994 [1987], Tome vı, p. 169-176. pour le gouvernement en pleine réforme de Napoléon III de marquer les hauts faits de la puissance française, et les succès de sa médecine sont à revendiquer comme des accomplissements de la nation. ${ }^{42}$ Grâce à l'aliénisme, spécialisation médicale qui a su se légitimer, la France et l'Empereur se portent garants des progrès de leur civilisation en matière de science et d'humanisme.

Tout en accomplissant cette œuvre de bienfaisance publique, destination essentielle de la Maison impériale de Charenton, l'État, dans sa munificence, s'est proposé un but plus large et plus élevé qui justifie complètement la grandeur de ses sacrifices pour la fondation de cette institution. Ce but, c'était la création d'un établissement qui pût servir de modèle, et qui fût, par son organisation administrative et médicale, l'image la plus parfaite des progrès atteints par la psychiatrie dans notre pays. ${ }^{43}$

Dans cette effervescence autour de la reconnaissance de la psychiatrie, les Monomanes de Géricault rappellent les moments fondateurs de ce qui est devenu une fierté nationale. Mais en 1863 , leur réalisme poignant répond aussi au goût en vogue alors que la monomanie est devenue un terme populaire. En nommant monomanes les portraits de ces cinq individualités, qui malgré les nombreuses spéculations, demeurent inconnues, la gloire de Géricault peut briller de tous ses feux. Les œuvres de Géricault deviennent signes d'une époque historique de la psychiatrie et ajoutent un fleuron supplémentaire à la gloire de l'artiste. Leur double destin est alors fixé: elles peuvent satisfaire un public élargi, tant de médecins que de collectionneurs d'art. Depuis lors, les spécialistes, commentateurs et autres experts se disputent sur la destination et la fonction de ces œuvres.

La vente des cinq œuvres en tant que groupe, souhaitée par Viardot dans sa lettre publiée dans la Gazette des Beaux-arts, n'eut pas lieu. ${ }^{44}$ Elles sont maintenant les fières possessions de cinq musées différents: en France (Paris, Monomane du jeu et Lyon, Monomane de l'envie), en Belgique (Gand, Monomane du vol), en Suisse (Winterthur, Monomane du commandement militaire) et aux États-Unis (Springfield, Monomane du vol d'enfants). Elles sont reproduites tant et plus dans les histoires générales de l'art, dans les monographies de l'artiste, dans les ouvrages et articles sur la psychiatrie, sur la physiognomonie, sur le portrait. La dualité qui leur a été attribuée par leurs premiers récepteurs en 1863 , Lachèze, Viardot et Blanc, se perpétue encore aujourd'hui, dans le discours d'une histoire de l'art qui reconduit aussi la légende de Géricault et qui sert le mythe de l'artiste rebelle. 